\title{
Nano-Sized Albumin-Copolymer Micelles for Efficient Doxorubicin Delivery
}

\author{
Yuzhou Wu • Er Kai Shih • Arvind Ramanathan • \\ Subhash Vasudevan · Tanja Weil
}

Received: 2 October 2011 / Accepted: 18 November 2011/Published online: 9 February 2012

(c) The Author(s) 2012. This article is published with open access at Springerlink.com

\begin{abstract}
We present the discovery of a nano-sized protein-derived micellar drug delivery system based on the polycationic albumin precursor protein cBSA-147. The anticancer drug doxorubicin (DOX) was efficiently encapsulated into nanosized micelles based on hydrophobic interactions with the polypeptide scaffold. These micelles revealed attractive stabilities in various physiological buffers and a wide $\mathrm{pH}$ range as well as very efficient uptake into A549 cells after $1 \mathrm{~h}$ incubation time only. In vitro cytotoxicity was five-times increased compared to free DOX also indicating efficient intracellular drug release. In addition, multiple functional groups are available for further chemical modifications. Based on the hydrophobic loading mechanism, various classical anti-cancer drugs, in principle, could be delivered even synergistically in a single micelle. Considering these aspects, this denatured albumin-based drug delivery system represents a highly attractive platform for nanomedicine approaches towards cancer therapy.
\end{abstract}

This article is part of the Topical Collection "In Focus: Nanomedicine".

Y. Wu $\cdot$ T. Weil $(\bowtie)$

Institute of Organic Chemistry III, Macromolecular Chemistry,

Albert-Einstein-Allee 11, 89081 Ulm, Germany

e-mail: Tanja.Weil@uni-ulm.de

Y. Wu $\cdot$ E. K. Shih $\cdot$ T. Weil

Department of Chemistry, National University of Singapore,

3 Science Drive 3, Singapore 117543, Singapore

A. Ramanathan $\cdot$ S. Vasudevan

Duke-NUS Graduate Medical School,

8 College Road, Singapore 169857, Singapore

\section{Introduction}

Nanomedicine has emerged as an innovative strategy for cancer therapy with great promise for clinical applications [1]. Nanomedical approaches to drug delivery involve the development of nanoscale particles or macromolecules to improve the biological activity and pharmacokinetic profile of drug candidates [1]. In particular, small-molecule chemotherapeutics often elicit severe side effects, such as anemia, vomiting, diarrhea, decreased immunity and alopecia, which is due to the systemic distribution of cytotoxic drugs [2]. In recent years, a large number of natural and synthetic macromolecules as well as inorganic particles have been developed as drug delivery nanocarriers [3], such as liposomes [4-6], polymeric nanoparticles [7, 8], inorganic nanoparticles [9] as well as proteins [10-13]. The long systemic circulation times of nano-sized drugs and drug carriers and the increased vascular permeability of tumor tissue leads to an accumulation at tumor sites, which is often referred to as passive targeting or "enhanced permeability and retention effect" (EPR effect) $[14,15]$. As a particular feature, such nano-sized drug delivery systems could serve as platform to integrate multiple additional functionalities such as specific targeting ligands or probes suitable for tumor imaging [16, 17]. Still, there are major challenges associated to the design of an ideal macromolecular drug delivery system [2,3]. In order to reduce drugunrelated side-effects in humans, biocompatibility of the entire drug delivery complex represents a key concern to achieve a suitable therapeutic window. In addition, the prevention of drug leakage during blood circulation on the one hand but achieving efficient drug release at target cells on the other hand represent crucial factors that still appear challenging to achieve. Other prerequisites include efficient permeability through vascular barriers and cell membranes, 
great specificity for tumor tissue, high loading of the drug cargo as well as fast degradation of the entire carrier system after delivery producing non-toxic metabolites. In view of these criteria, it is not astonishing that only few drug delivery systems have entered clinical trials yet.

Doxorubicin (DOX) represents a potent cytotoxic drug that has been applied to address a broad number of different kinds of cancers [18, 19]. It consists of an anthracycline antibiotic DNA intercalator inhibiting DNA replication, which has been successfully applied in cancer chemotherapy $[18,19]$. For solubility reasons, DOX needs to be administered intravenously as hydrochloride salt, which limits its cellular uptake and cardiac toxicity due to unspecific cell uptake represents a serious side-effect [20]. Therefore, liposome formulations such as Doxil ${ }^{\mathrm{TM}}$ containing DOX have entered the market [21]. However, inherent limitations of liposomes such as drug leakage into the plasma and vascular capillaries still leaves room for improvement $[22,23]$. In addition, dendrimers have been applied successfully for the complexation of DOX [24]. Dendrimer mediated complexation is advantageous in terms of stability, controlled release, drug payload and reduced toxicity of the entrapped drug(s) [25]. However, even though studies on non-covalent interactions of dendrimers with drugs suggest improved drug solubilization, low numbers of encapsulated drug molecules and limited complex stabilities represent key concerns [25].

Protein-based drug delivery systems have emerged recently since they consist of "natural", non-toxic monomers, they reveal low cytotoxicity and particularly human serum albumin (HSA) is known to exhibit insignificant immunogenicity [11]. HSA is a well-known transportation protein ubiquitous in the blood plasma to allow the delivery of hydrophobic nutrients [26]. Utilizing its native transportation mechanism, albumin formulated cancer drugs, such as Abraxane ${ }^{\mathrm{TM}}$ (albumin formulated paclitaxel for treating breast cancer), have been successfully introduced to the market and show higher efficiencies in patients without prominent side-effects such as allergic reactions [27-29]. However, in general, protein-based delivery platforms also bear several draw-backs such as their low membrane permeability, immunogenicity and low metabolic stability.

Herein, we disclose a novel type of denatured albuminbased copolymer that facilitates encapsulating hydrophobic drug molecules thus forming nano-sized, stable micelles that are able to efficiently pass cell membranes. Recently, protein-derived copolymers were introduced, consisting a polypeptide backbone and several grafted, hydrophilic polyethylene(oxide) (PEO) side chains (Wu et al., in preparation, [30-32]). PEO side-chains are known to reduce non-specific interactions as well as immunogenicity and antigenicity of proteins and peptides. The promising applications of such copolymers include surface patterning
(Wu et al., in preparation), nanoparticles coating [30], gene delivery [31] as well as targeted delivery of hydrophobic molecules into cancer cells [32]. Such biohybrid micelles represent a novel type of delivery platform offering great potential to effectively transport and release lipophilic drugs into cancer cells.

\section{Experimental}

\subsection{Materials}

Albumins from bovine serum (BSA) $(\geq 98 \%$, Sigma Aldrich), $O$-(2-maleimidoethyl)- $O^{\prime}$-methyl-polyethylene glycol 5000 (PEG-5000-MI) (>90\% NMR, Aldrich), propargylamine (98\%, Aldrich), maleic anhydride ( $\geq 99.0 \%$, Fluka), glacial acetic acid (ACS 99.7\%, Alfa Aesar), $\mathrm{N}$-hydroxysuccinimide (98\%, Aldrich), tris(2-carboxyethyl)phosphine hydrochloride (TCEP) (98\%, Alfa Aesar), Urea (98+\%, Alfa Aesar), ethylenediaminetetraacetic acid (EDTA) (99\%, Alfa Aesar) and Trizma ${ }^{\mathrm{TM}}$ base (BioUltra, $\geq 99.8 \%$, Sigma), doxorubicin hydrochloride salt (DOX) ( $\geq 99.0 \%$, XingCheng ChemPhar Co. LTD, China) were used as received without further purification. Bio-Rad BioGel P30 was used for desalting, vivaspin ultrafiltration tubes were purchased from GE healthcare for purification. Dulbecco's Modified Eagle Medium (DMEM) (1X) liquid (high glucose), Fetal Bovine Serum (FBS) Standard Quality (EU approved), penicillin/streptomycin solution $(100 \times)$ were purchased from PAA Laboratories $\mathrm{GmbH}$, MEM [non-essential amino acids solution $10 \mathrm{mM}(100 \times)$ ] was purchased from Invitrogen.

\subsection{Synthesis of $N$-Propargyl Maleimide}

Maleic anhydride $(980 \mathrm{mg}, 10 \mathrm{mmol})$ was reacted with propargylamine $(550 \mathrm{mg}, 10 \mathrm{mmol})$ in $20 \mathrm{~mL}$ glacial acetic acid at room temperature (RT) overnight. Then, sodium acetate $(164 \mathrm{mg}, 2 \mathrm{mmol})$ and acetic anhydride $(10 \mathrm{~mL})$ were added and the reaction mixture was heated to $65^{\circ} \mathrm{C}$ for $2 \mathrm{~h}$. After reaction, all solvent was removed under vacuum and the residue was purified by silica gel column chromatography (EtOAc:Hexane 1:3) to yield $60 \mathrm{mg}$ of a white solid (yield 5\%). Rf 0.56 (EtOAc/heptane, 1:1 v/v), ${ }^{1} \mathrm{H}$ NMR (CDCl3, $300 \mathrm{MHz}): \delta 6.75$ (s, 2H), 4.28 (d, 2H, J $1.8 \mathrm{~Hz}$,); $2.20(\mathrm{t}, 1 \mathrm{H}, \mathrm{J} 1.8 \mathrm{~Hz}) ;{ }^{13} \mathrm{CNMR}\left(\mathrm{CDCl}_{3}, 300 \mathrm{MHz}\right): \delta 169.2$, 134.4, 76.9, 71.5, 26.8. MS-ESI, $136 \mathrm{M}+$.

\subsection{Preparation of cBSA-147-PEO $(5000)_{28}$ (2)}

dcBSA-147-PEO(5000) 28 (2) was prepared using a similar procedure reported in our previous paper [30]. Briefly, cationic bovine serum albumin, cBSA-147 [33] (1, $10 \mathrm{mg}$, 
$0.15 \mu \mathrm{mol})$ was first denatured in degassed urea-phosphate buffer $(10 \mathrm{~mL}, 10 \mathrm{mM}$ phosphate buffer, $5 \mathrm{M}$ urea and 2 mM EDTA, pH 7.4) for $10 \mathrm{~min}$, and then reducing agent TCEP $(4.3 \mathrm{mg}, 15 \mu \mathrm{mol})$ was added under argon atmosphere for $30 \mathrm{~min}$. Subsequently, PEG-5000-MI $(77 \mathrm{mg}$, $15 \mu \mathrm{mol})$ was added to the reaction and stir at RT for $3 \mathrm{~h}$. Finally, the capping reagent $\mathrm{N}$-propargyl maleimide $(30 \mu \mathrm{mol})$ was given to the reaction and stir for another $3 \mathrm{~h}$. The reaction mixture was first purified by ultrafiltration with Tris- $\mathrm{HCl}$ buffer $(20 \mathrm{mM}$ Tris, $150 \mathrm{mM} \mathrm{NaCl}$, $2 \mathrm{mM}$ EDTA, $\mathrm{pH}$ 7.4), and followed by further size exclusion purification using HiPrep ${ }^{\mathrm{TM}}$ Sephacyl $^{\mathrm{TM}}$ S-100 HR gel filtration column on AKTÄ Purifier flash protein liquid chromatography with Tris- $\mathrm{HCl}$ buffer $(20 \mathrm{mM}$ Tris, $150 \mathrm{mM} \mathrm{NaCl}, \mathrm{pH}$ 7.4). Then, the purified material was desalted and lyophilized to yield dcBSA-147-PEO $(5000)_{28}$ (2) as white fluffy solid. The product was characterized by gel electrophoresis using precast NuPAGE TA 3-8\% Gel (Invitrogen) in Novek ${ }^{\mathrm{TM}}$ Mini-Cell.

\subsection{Preparation of dcBSA-147-PEO $(5000)_{28}$ Micelle (3)}

DOX hydrochloride $(\mathrm{DOX} \times \mathrm{HCl})(4 \mathrm{mg}, 7 \mathrm{mmol})$ was dissolved in $500 \mu \mathrm{L}$ deionized-distilled water and mixed with 1.2 equivalents of triethylamine $(1.2 \mu \mathrm{L}, 8.4 \mathrm{mmol})$. The aqueous solution was extracted five times with dichloromethane. The combined organic extracts were evaporated in vacuum and $3 \mathrm{mg}$ of DOX were obtained. This DOX stock solution was prepared by dissolving the obtained solid in $500 \mu \mathrm{L}$ of DMF to achieve a total concentration of $6 \mathrm{mg} / \mathrm{mL}$. dcBSA-147-PEO $(5000)_{28}$ (2) $(0.5 \mathrm{mg}, 0.0028 \mu \mathrm{mol})$ in distilled-deionized water was combined with the desired molar ratios of DOX, (ratios of $1: 1,1: 5,1: 10,1: 20,1: 50,1: 100)$ and allowed to stir overnight in the dark at RT. The reaction mixtures were transferred to 3.5 K MWCO Slide-A-Lyzer MINI Dialysis cassettes (Pierce) and dialyzed in $1 \mathrm{~L}$ distilled-deionized water for $24 \mathrm{~h}$ at $4^{\circ} \mathrm{C}$; during this time period, water was exchanged 3-5 times. The amount of entrapped DOX in each molar ratio was determined by measuring the absorbance at $488 \mathrm{~nm}$ using Tecan M-1000 microplate reader.

\subsection{Negative Staining Transmission Electron Microscopy}

The morphology of dcBSA-147-PEO(5000) $)_{28}-(\text { DOX })_{14}$ micelles was characterized via TEM applying the negative staining technique. A drop of $1 \mathrm{mg} / \mathrm{mL}$ dcBSA-147$\mathrm{PEO}(5000)_{28}-(\mathrm{DOX})_{14}$, dissolved in an aqueous solution was deposited onto a hydrophilic copper grid with a carbon film for approximately $1 \mathrm{~min}$ and then blotted excess of the sample by filter paper. The grid was allowed to dry at RT for overnight and then stained with $1 \%$ Uranyl acetate solution for TEM investigations.

\subsection{Dynamic Light Scattering}

The hydrodynamic size distribution of dcBSA-147PEO $(5000)_{28}-(\mathrm{DOX})_{14}$ micelles was characterized by DLS using a Malvern Nanosizer (Malvern Ltd, Malvern, UK). dcBSA-147-PEO $(5000)_{28}-(\text { DOX })_{14}$ was prepared at $0.15 \mathrm{mg} / \mathrm{mL}$ concentration in aqueous solution and filtered through $0.2 \mu \mathrm{m}$ microsyringe filter before measurement to avoid dust contamination. Autocorrelation functions were analyzed by cumulants method and COTIN routine to estimate hydrodynamic diameter. The hydrodynamic diameter distribution was presented as number distribution.

\subsection{Characterization of Micelle Stability in Various Media}

$100 \mu \mathrm{L}$ aliquots of $0.1 \mathrm{mg}$ dcBSA-147-PEO(5000) $)_{28^{-}}$ $(\mathrm{DOX})_{14}$ samples were dialyzed using 3.5 K MWCO Slide-ALyzer MINI Dialysis devices (Pierce) in $10 \mathrm{mM}$ pH 3 and 5 citrate buffer and $10 \mathrm{mM}$ (pH 7 and 9) and Tris buffer, respectively, for 2 days and the absorbance spectra were recorded before and after dialysis.

\subsection{Cell Culture}

Hela cells (human cervix carcinomic cell line) and A549 cells (carcinomic human alveolar basal epithelial cell line) were obtained from DSMZ (German Collection of Microorganisms and Cell Cultures, Braunschweig) and cultured in DMEM medium with high glucose supplemented and with $10 \%$ fetal bovine serum (FBS), $100 \mathrm{U} / \mathrm{mL}$ Penicillin, $0.1 \mathrm{mg} / \mathrm{mL}$ Streptomycin, $0.1 \mathrm{mM}$ non-essential amino acids at $37^{\circ} \mathrm{C}$ in a humidified $5 \% \mathrm{CO}_{2}$ incubator.

\subsection{Cytotoxicity Assay}

HeLa cells were plated into a white 96 well microplate at a density of 8000 cells per well and incubated overnight for attachment. After incubation, the media from each well were aspirated and exchanged with $100 \mu \mathrm{L}$ of fresh DMEM medium and the desired amount of either free DOX or dcBSA-147-PEO(5000) $)_{28}-(\mathrm{DOX})_{14}$ micelles. All concentrations were prepared as triplicates. After further incubation with drug molecules for 12, 24 and $48 \mathrm{~h}$, cell viability was tested by Cell-titer-Glo ${ }^{\mathrm{TM}}$ (Promega) cell viability assay kit according to manufactory's instruction. Cells that were not treated with any drug were considered as blank. The $\mathrm{IC}_{50}$ values and the $50 \%$ inhibitory concentrations were obtained through GraphPad Prism 3 software. 
2.10 Live Cell Imaging of Intracellular DOX in A549 Cells

A549 cells were plated onto glass cover slips and incubated for $12 \mathrm{~h}$ to allow cell attachment and spreading. Thereafter, $0.1 \mu \mathrm{M}$ of either free DOX or dcBSA-147-PEO $(5000)_{28^{-}}$ (DOX)14 micelles were added to the cells and incubated for one additional hour. The cells were then washed 3 times with PBS, replaced with fresh medium and incubated with $5 \mu \mathrm{g} / \mathrm{ml}$ of WGA-Alexa Fluor 594 conjugate (Cat \# $\mathrm{W} 11262$, Invitrogen) for $10 \mathrm{~min}$ to label the cell membrane and imaged without further washing. Microscopy was performed with a Zeiss LSM 710 META laser scanning microscope fitted with Argon, HeNe543 and Diode405-30 lasers. Z-sections of chosen field were acquired with a $40 \times$ oil immersion objective (Zeiss, Germany). Excitation and emission wavelengths of 535-562 nm for DOX and of 600-620 nm for WGA-Alexa Fluor 594 detection were selected. The acquired images were processed with Zen software developed by Carl Zeiss.

\subsection{Quantification of DOX Uptake}

For the quantification of DOX cell uptake, images obtained from five different sections in each of the slides were analyzed using the public domain NIH ImageJ program with default settings (developed at the US National Institutes of Health and available on the Internet at http://rsb.info.nih.gov/nih-image/). To calculate the percentage of intracellular DOX, the fluorescence intensity of DOX [green] was divided by the total fluorescence (DOX [green] and the cell membrane [red]) and multiplied by 100.

\section{Results and Discussion}

\subsection{Preparation and Characterization of dcBSA-147-PEO(5000) ${ }_{28}$ DOX Micelles}

Polycationic bovine serum albumin (cBSA-147) was used as precursor protein for the preparation of the PEO-grafted copolymer dcBSA-147-PEO $(5000)_{28}$ (2). cBSA-147 stands for cationic BSA, which was obtained after converting about 87 out of 100 carboxylic acid groups of aspartic and glutamic acid residues of native BSA into primary amino groups according to published procedures [33]. cBSA-147 serves as efficient gene delivery carrier of low cytotoxicity that reveals fast and efficient cell uptake and even allows stable gene transfections [33] and it has been applied successfully as biocompatible surface coating [34]. However, due to the potential immunogenicity of cationic albumin derivatives [35], further modifications are required to efficiently shield epitopes to allow multiple dose applications in vivo. Therefore, cBSA-147 was converted into a polypeptide single chain copolymer (2) under controlled denaturing conditions ( $5 \mathrm{M}$ urea) and the presence of the mild reduction agent TCEP to destabilize the globular architecture of BSA and reduce all 17 disulfide bridges (Fig. 1). The denatured protein backbone was stabilized in situ by applying long, hydrophilic polyethyleneoxide (PEO-5000) side chains carrying a single thiol-reactive maleimide group yielding the PEO grafted copolymer dcBSA-147-PEO $(5000)_{28}$ in one reaction step. To contribute to the clarity of the nomenclature, dcBSA-147$\operatorname{PEO}(5000)_{28}$ denotes denatured cationic BSA-147 [31] carrying about 28 grafted PEO-5000 chains with a molecular weight of $5,000 \mathrm{~g} / \mathrm{mol}$. Thereafter, $N$-propargyl

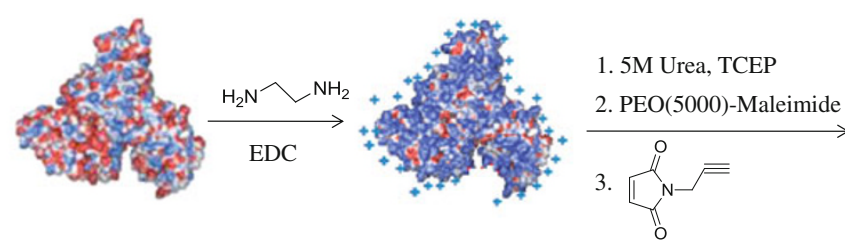

(1)

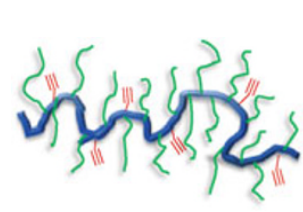

(2)

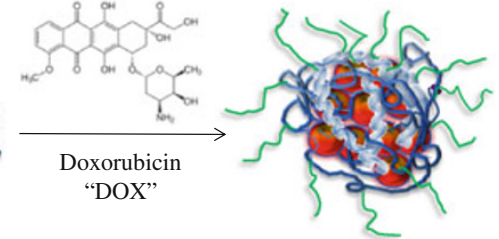

(3)

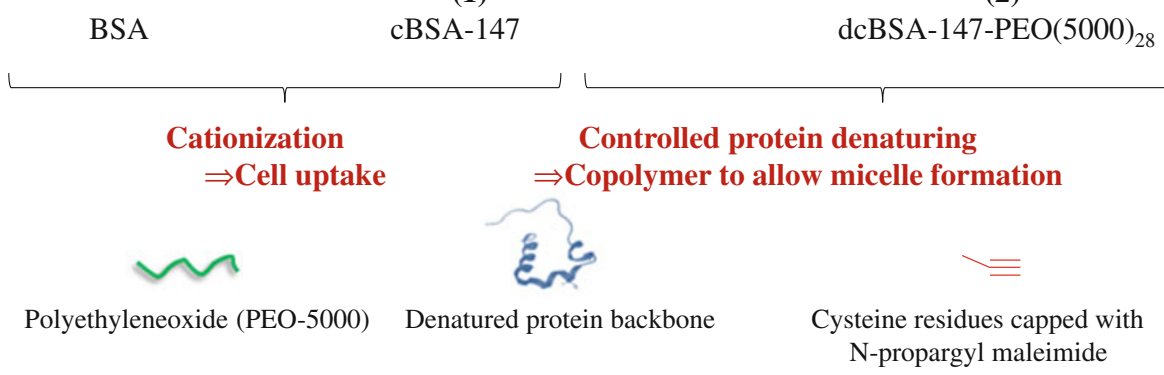

Fig. 1 Preparation of dcBSA-147-PEO(5000) ${ }_{28}$-DOX micelles 
Fig. 2 Characterization of dcBSA-147-PEO $(5000)_{28}$ (2) and dcBSA-147-PEO(5000) ${ }_{28}^{-}$ $(\mathrm{DOX})_{14}$ micelles. a SDSPAGE of cBSA-147 (1) (line 1) and dcBSA-147-PEO(5000) 28 (2) (line 2); b agarose gel electrophoresis of dcBSA-147$\operatorname{PEO}(5000)_{28^{-}}(\mathrm{DOX})_{14}$ micelles in TAE buffer (Tris base-acetic acid-EDTA buffer, pH 7.4); c loading of $\mathrm{DOX} \mathrm{HCl}$ and dcBSA-147-PEO(5000) ${ }_{28}$ $(\mathrm{DOX})_{14}$ micelles onto a Biogel P30 size exclusion column using the same amount of eluent (a)

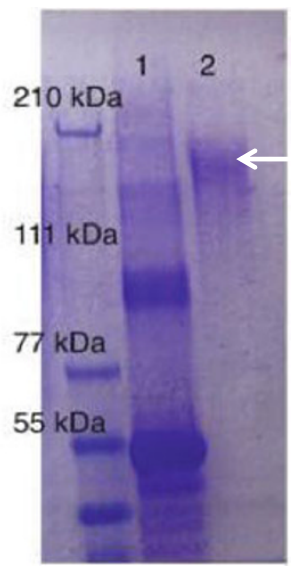

(b)

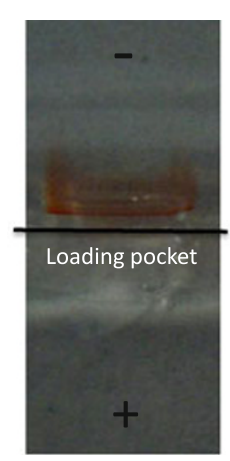

(c)

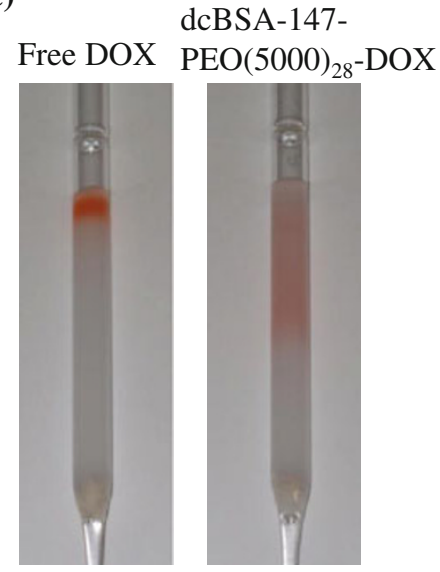

maleimide was added to cap all remaining free thiol groups that have not reacted with the PEO chains for steric reasons. This capping step is essential and significantly improves the shelf life the denatured PEO-grafted copolymers [30]. In addition, the $N$-propargyl moiety further contributes to the lipophilicity of the polypeptide backbone which is crucial for micelle formation in aqueous solution. Denaturation of globular BSA exposes a large number of functional groups along the backbone, which were previously hidden inside the protein scaffold and become available for further reactions. In addition, lipophilic amino acids originating from e.g. hydrophobic pockets are now accessible and, together with the $N$-propargyl groups, allow efficient interaction with hydrophobic drug molecules. The presence of multiple positive charges contribute to increased membrane permeability and cellular uptake and the high number of PEO side chains distributed along the backbone were attached to reduce nonspecific adsorption and to minimize immunogenicity. According to SDS-PAGE, dcBSA-147$\operatorname{PEO}(5000)_{28}$ (2) prepared herein possesses an approximate molecular weight of about $200 \mathrm{kDa}$ (Fig. 2a). Considering the presence of multiple functional groups along the polypeptide backbone corresponding to the peptide sequence of BSA (by considering that most carboxylic acids have been converted into amino groups), this grafted polymer represents an attractive platform for conjugating targeting ligands and other functional moieties.

Serum albumin transports water-insoluble lipids in the bloodstream and about six hydrophobic ligands could be transported [36]. After denaturation, the tertiary structure of dcBSA-147-PEO $(5000)_{28}$ has been destroyed but sufficient numbers of lipophilic amino acids and additional $N$-propargylamine are still present that could non-covalently interact with hydrophobic molecules. $\mathrm{DOX} \mathrm{HCl}$ was chosen as model drug since it has been well characterized in vitro and in vivo, it has a lipophilic scaffold and emits above $500 \mathrm{~nm}$ thus facilitating characterization via fluorescence microscopy. DOX loading was performed according to published procedures [37]. Briefly, DOX hydrochloride salt was first neutralized and extracted into a dichloromethane layer. The obtained non-water soluble drug was then re-dissolved in a minimum amount of dimethylformamide and added into dcBSA-147-PEO $(5000)_{28}(2,1 \mathrm{mg} / \mathrm{mL})$ dissolved in aqueous solution. After overnight stirring, micelles were purified by dialysis. The purification efficiency was accessed by loading DOX micelles (3) and free DOX $\mathrm{HCl}$ into Bio-Gel P30 (Bio-Rad) size exclusion columns. Free DOX $\mathrm{HCl}$ is indicated by a red colored narrow band, which remains on top of the column, whereas sufficiently stable DOX micelles quickly moved along the column and no traces of free DOX were present on top of the column suggesting that all DOXs were associated with the copolymer micelles (Fig. 2c).

\subsection{Characterization of dcBSA-147-PEO $(5000)_{28}$ DOX Micelles}

In order to quantify the average number of encapsulated DOX molecules as well as the maximum drug loading efficiency of the micelle system, different equivalents of DOX were applied for micelle preparation. After dialysis, the absorbance spectra of the micelles were recorded (Fig. 3a) and the characteristic DOX absorbance with a maximum at $488 \mathrm{~nm}$ was observed indicating successful DOX loading. The DOX concentration was calculated considering a molar extinction coefficient of $11,500 / \mathrm{M} / \mathrm{cm}$ and the average number of DOX encapsulated in the micelles is given in Table 1. DOX loading could be estimated due to a linear correlation of the amount of DOX added in access to the copolymer solution (feed DOX) versus the number of encapsulated DOX molecules (Fig. 3b). By adding 100 equivalent of DOX, a maximum number of about 14 DOX molecules was reproducibly encapsulated by each micelle. In contrast, the native 

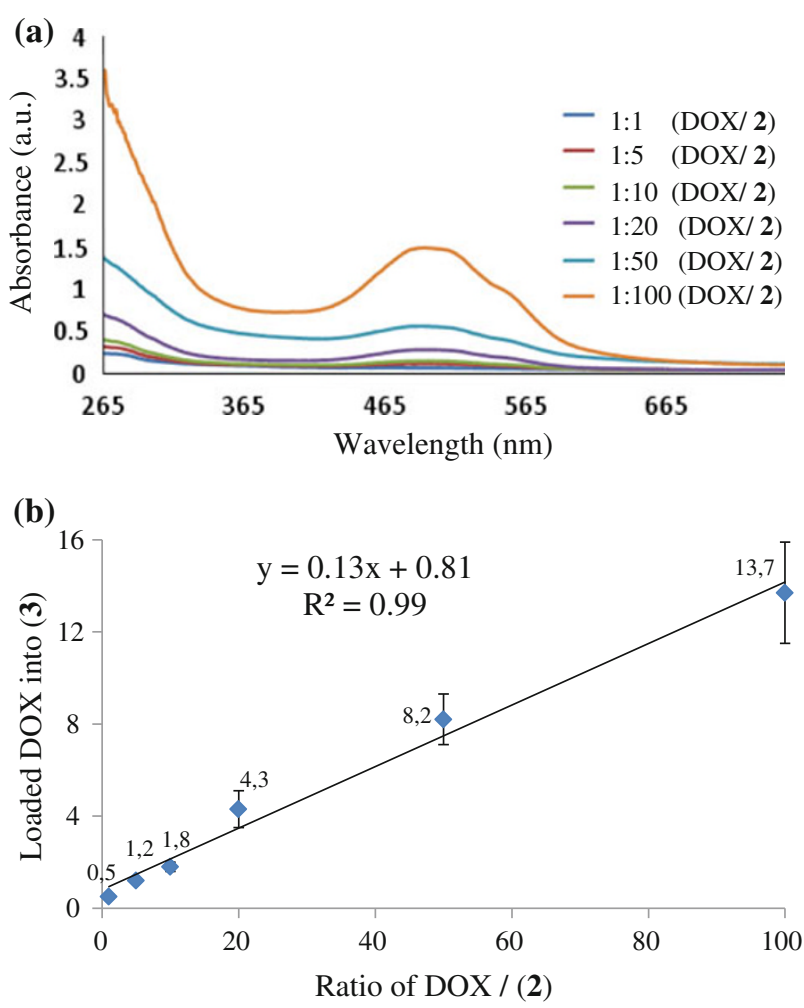

Fig. 3 Loading of different ratio of DOX into dcBSA-147PEO $(5000)_{28}-(\mathrm{DOX})_{14}$ micelles (3). a Absorbance spectra of dcBSA-147-PEO (5000) ${ }_{28}$-DOX for the same concentrations of (2) but different concentrations of DOX loaded into dcBSA-147PEO $(5000)_{28}$ micelles (3); $\mathbf{b}$ correlation of the ratio of added DOX (feed DOX) versus (2) in solution and the amount of DOX loaded into micelles (3)

Table 1 Summary of the loading efficiency of DOX molecules into dcBSA-147-PEO $(5000)_{28}$ micelles

\begin{tabular}{|c|c|c|c|}
\hline $\begin{array}{l}\text { Ratio } \\
\text { added } \\
\operatorname{DOX} /(2)^{\mathrm{a}}\end{array}$ & $\begin{array}{l}\text { Loading } \\
\text { efficiency } \\
\text { (Molar ratio) }^{\text {b }}\end{array}$ & $\begin{array}{l}\text { Loading } \\
\text { efficiency } \\
{\text { (in } w t \%)^{c}}^{c}\end{array}$ & $\begin{array}{l}\text { Encapsulation } \\
\text { efficiency } \\
\text { of DOX }(\%)^{\mathrm{d}}\end{array}$ \\
\hline $1: 1$ & $0.5 \pm 0.1$ & $0.15 \pm 0.03$ & $51.6 \pm 9.9$ \\
\hline $5: 1$ & $1.2 \pm 0.1$ & $0.34 \pm 0.01$ & $23.1 \pm 1.0$ \\
\hline $10: 1$ & $1.8 \pm 0.2$ & $0.52 \pm 0.07$ & $17.2 \pm 2.3$ \\
\hline $20: 1$ & $4.3 \pm 0.8$ & $1.22 \pm 0.23$ & $20.4 \pm 3.9$ \\
\hline $50: 1$ & $8.2 \pm 1.1$ & $2.33 \pm 0.31$ & $15.5 \pm 2.0$ \\
\hline 100:1 & $13.7 \pm 2.2$ & $3.91 \pm 0.63$ & $13.1 \pm 2.1$ \\
\hline
\end{tabular}

${ }^{a}$ Molar ratio of feed DOX added versus dcBSA-147-PEO(5000) ${ }_{28}$ (2) for micelle formation

b Molar ratio of DOX loaded into dcBSA-147-PEO(5000) ${ }_{28}$ micelles

c Weight percentage of DOX loaded into dcBSA-147-PEO(5000) ${ }_{28}$ micelles

${ }^{d}$ Weight percentage of loaded DOX to added DOX (feed DOX)

protein carrier HSA is only able to accommodate up to six lipophilic guest molecules [36] thus indicating an increased drug load after protein denaturation. The morphology and size distribution of micelles containing about 14 DOX were investigated by TEM with uranyl acetate staining. Very homogenous micelles with narrow size-distributions were observed by TEM. Their size with average diameters of about $56 \mathrm{~nm}$ (Fig. 4a, b) is generally considered an ideal size for passive targeting and accumulation into tumor tissues by the ERP effect $[14,15]$. The size distribution of dcBSA-147-PEO $(5000)_{28}-(\mathrm{DOX})_{14}$ micelles in solution was further investigated using DLS and narrowly dispersed particles were found with average diameter of approximately $30 \mathrm{~nm}$ which is comparable with the particle size found by TEM. In addition, DOX-containing micelles were characterized by a highly positive surface charge originating from the cationic nature of the cBSA-147 precursor protein, which was confirmed by gel electrophoresis (Fig. 2b) and which ensures electrostatic interaction with cellular membranes and cell uptake.

\subsection{In Vitro Assessment of Micelle Stability}

Undesired drug leakage in blood plasma represents a common limitation for most drug deliver carriers, which is known to cause several serious side effects. To study the stability of (3) in different media, dcBSA-147-PEO(5000) $)_{28^{-}}(\mathrm{DOX})_{14}$ micelles containing about 14 DOX molecules were dialyzed in PBS buffer as well as DMEM cell culture medium for 4 days. The absorbance spectra were recorded before and after dialysis (Fig. 5a). It is noteworthy that no changes of the absorbance occurred indicating high micelle stability under high ion strength and cell culture conditions. Furthermore, the stability of these micelles at different $\mathrm{pH}$ was also investigated. Dialysis was performed in the physiological relevant range from $\mathrm{pH} 3$ to $\mathrm{pH} 9$ for 2 days, and the absorbance spectra of these micelles did not reveal any changes (Fig. 5b). Therefore, the albumin-derived copolymer micelles display remarkably high stabilities in solution and no drug leakage was observed which is very critical for drug delivery applications and might contribute to an improved side effect profile. The notably stability could attribute to multivalent interactions of protein hydrophobic moieties with DOX molecules, which is especially attractive comparing to the liposome formulated Doxil ${ }^{\mathrm{TM}}$ using in the market.

\subsection{Enhanced Cellular Uptake}

The unfavorable cellular uptake efficiency of DOX is known as one of the major limitations of DOX chemotherapy [20]. The stable encapsulation of DOX into positively charged albumin micelles should facilitate cellular uptake via endocytosis pathways. Therefore, the cellular uptake efficiency of dcBSA-147-PEO(5000) $)_{28}$ (DOX) 14 micelles into A549 cells was followed by confocal microscopy (Fig. 6). Adenocarcinomic human alveolar 
Fig. 4 a Negative staining TEM imaging of dcBSA-147$\operatorname{PEO}(5000)_{28}-(\mathrm{DOX})_{14}$; b size distribution of dcBSA-147PEO $(5000)_{28}-(\text { DOX })_{14}$ micelles calculated by the program Image J (a)

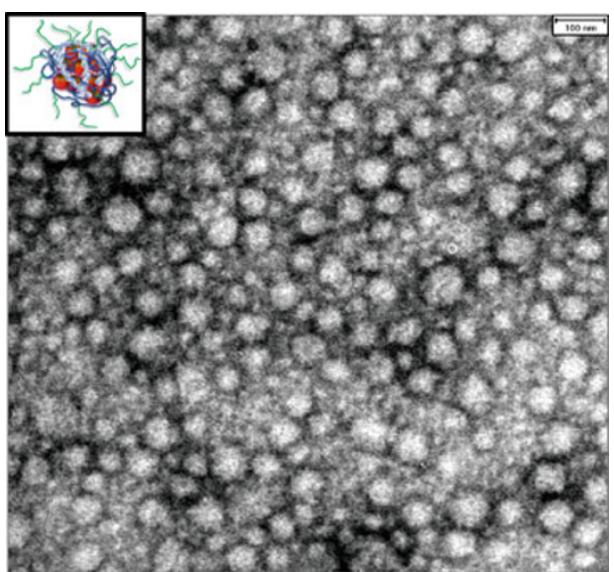

(b)

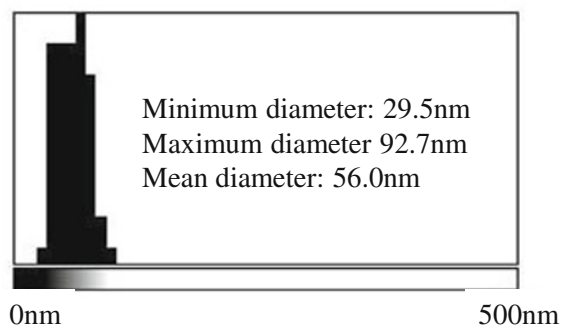

Fig. 5 Size distribution of dcBSA-147-PEO(5000) ${ }_{28}{ }^{-}$ $(\mathrm{DOX})_{14}$ micelles measured by DLS
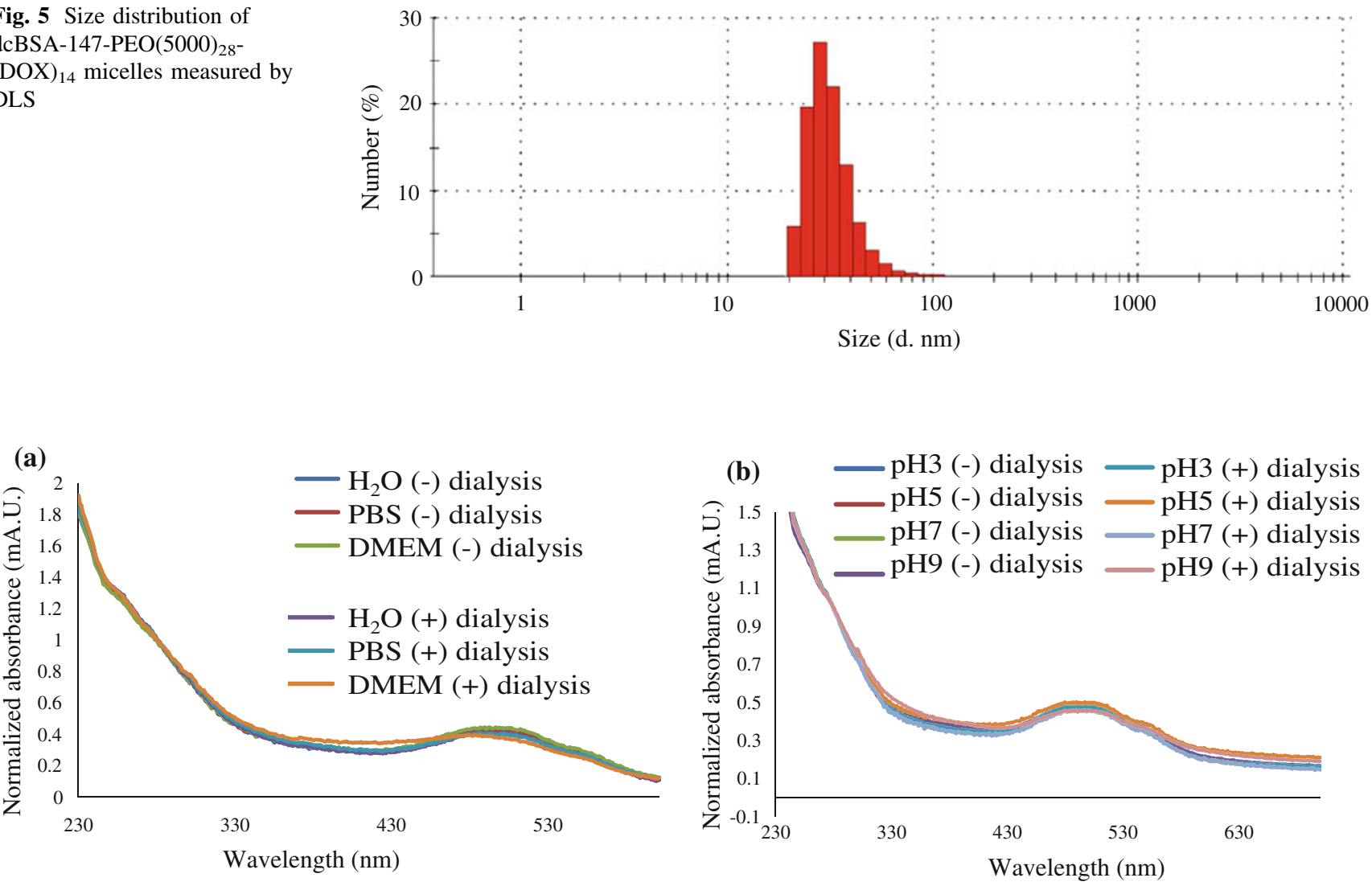

Fig. 6 a Absorbance spectra of dcBSA-147-PEO $(5000)_{28}-(\mathrm{DOX})_{14}$ micelles before and after dialysis in different physiological buffers; b absorbance spectra of dcBSA-147-PEO $(5000)_{28}-(\mathrm{DOX})_{14}$ micelles before and after dialysis in different $\mathrm{pH}$ buffer solutions

basal epithelial cells (A549 cells) were used to assess cellular permeability into epithelial tissue. After $1 \mathrm{~h}$ only, significantly increased cellular uptake of dcBSA-147$\mathrm{PEO}(5000)_{28}-(\mathrm{DOX})_{14}$ at $0.1 \mu \mathrm{M}$ concentration was observed. In addition, free DOX hydrochloride salt of the same concentration was applied in parallel as comparison. As shown in Fig. 6, substantial amount of DOX (green channel) was found in such cells treated with DOX micelle
(3), whereas considerably less emission intensity was collected for such cells incubated with non-complexed, free DOX molecules. The quantification of DOX uptake was calculated based on these confocal micrographs (Fig. 7). DOX micelles revealed more than 5 times higher uptake efficiency compared to free DOX, which is also reflected by the difference in cytotoxicity of both samples as discussed below. 

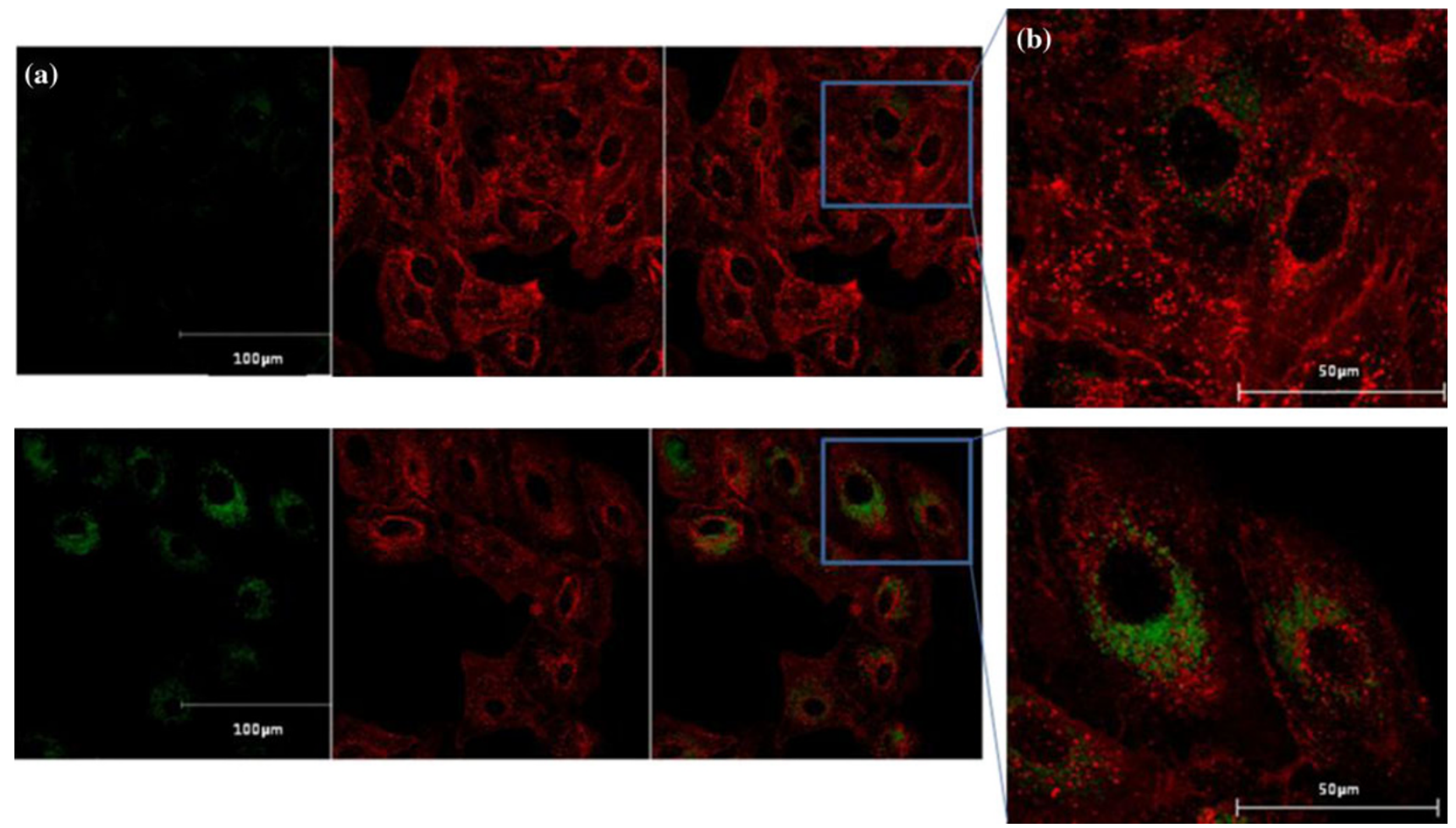

Fig. 7 Live confocal images of DOX in A549 cells. a DOX in dcBSA-147-PEO(5000) $)_{28}-(\mathrm{DOX})_{14}$ is delivered into the A549 cells, top panel cells incubated with free DOX, bottom panel A549 cells

\subsection{In Vitro Cytotoxicity of dcBSA-147-PEO $(5000)_{28^{-}}$ $(\mathrm{DOX})_{14}$}

Hela cells are considered the most common human carcinomic cell model used in cancer research. The cytotoxic activity of dcBSA-147-PEO(5000) $)_{28}-(\mathrm{DOX})_{14}$ micelles was evaluated in Hela cells and compared with DOX hydrochloride salt. Hela cells were treated with a series of concentrations of dcBSA-147-PEO $(5000)_{28}-(\mathrm{DOX})_{14}$ or the free drug $\mathrm{DOX} \mathrm{HCl}$ for 12, 24 and $48 \mathrm{~h}$ and cell viability at each concentration was tested by applying the Cell-TiterGlo cell viability assay (Promega) and plotted (Fig. 8). For all 3 incubation times, dcBSA-147-PEO(5000) $)_{28}$-(DOX) 14 exhibited an around five-times increased cytotoxicity compared to the DOX hydrochloride salt. After $12 \mathrm{~h}$ of incubation, an $\mathrm{IC}_{50}$ value of the DOX micelles of about $1.1 \pm$ $0.2 \mu \mathrm{M}$ was found (free DOX: $\mathrm{IC}_{50}$ of $5.5 \pm 0.7 \mu \mathrm{M}$ ), which was further decreased down to $0.4 \pm 0.01$ (free DOX: $\left.\mathrm{IC}_{50}=1.5 \pm 0.3 \mu \mathrm{M}\right)$ and $0.14 \mu \mathrm{M}$ after $48 \mathrm{~h}$ of incubation, indicating in all cases a five-times increased cytotoxicity of dcBSA-147-PEO(5000) $)_{28}$-(DOX) 14 micelles compared with free DOX $\mathrm{HCl}$ (Fig. 9). Extended observation times for achieving the maximum biological activity have been reported for other delivery systems as well [33] and are most likely due to slower cell uptake and drug release inside the cell. The attractive in vitro cytotoxic activity of dcBSA-147- incubated with dcBSA-147-PEO(5000) $)_{28}-(\mathrm{DOX})_{14}$. b Enlarged view of a field (marked by a square) from panel A. Cell membrane (red color), DOX (green color)

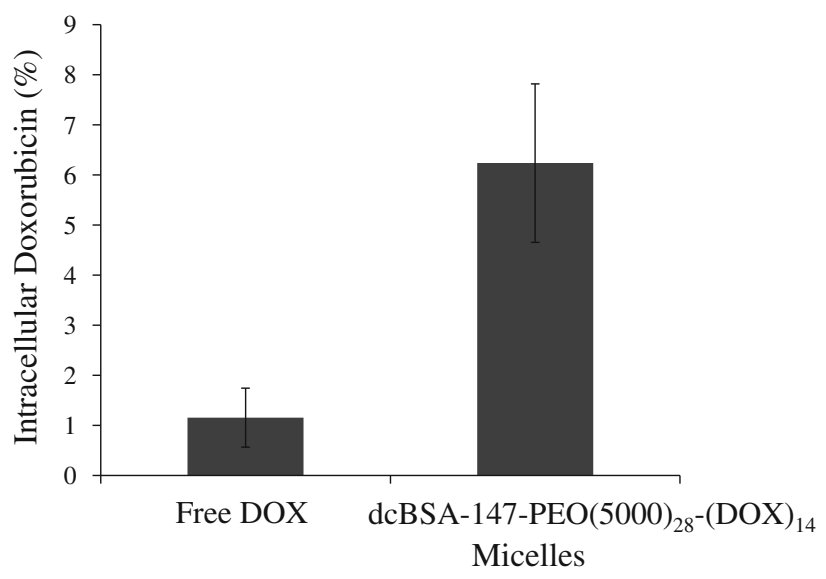

Fig. 8 Quantification of intracellular DOX in A549 cells after $1 \mathrm{~h}$ incubation time

PEO $(5000)_{28^{-}}(\mathrm{DOX})_{14}$ micelles could be attributed to their high membrane permeability benefits from multiple positive charges, as well as efficient carrier degradation and drug release in lysosomes.

\section{Summary and Conclusions}

The efficient protein-derived drug delivery system dcBSA$147-\mathrm{PEO}(5000)_{28}(2)$ was prepared based on the polycationic 


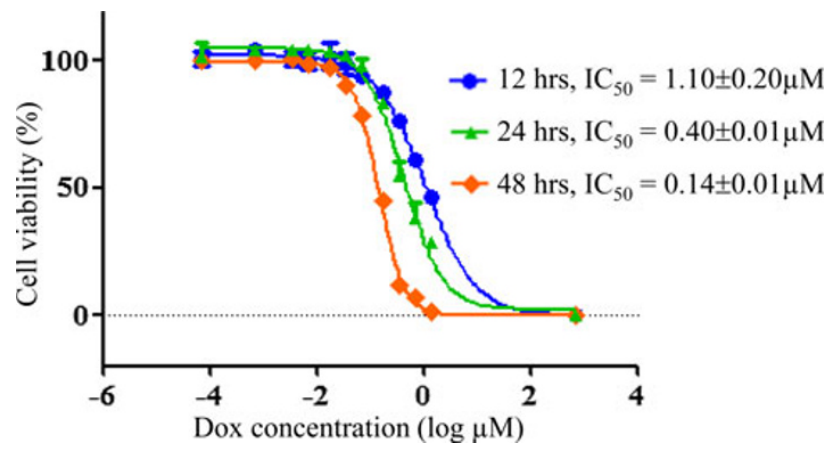

Fig. 9 Cytotoxicity of dcBSA-147-PEO $(5000)_{28}-(\mathrm{DOX})_{14}$ micelles after 12, 24 and $48 \mathrm{~h}$ incubation times

albumin precursor protein BSA-147. This copolymer was designed to encompass several important features such as (1) positive charges to facilitate cellular uptake via endocytosis, (2) a large number of hydrophilic and lipophilic groups along the backbone facilitating the formation of nanosized micelles of about $56 \mathrm{~nm}$ with narrow size distributions and high stability in different physiological buffers, cell media and a broad $\mathrm{pH}$ range, (3) about $28 \mathrm{PEO}$ chains along the backbone to reduce plasma protein binding, immunogenicity and contribute to high micelle stability in solution, (4) high drug loading of about 14 lipophilic DOX molecules per micelle. Due to the synergistic combination of all these features, unique nanosized containers were achieved that are attractive to efficiently encapsulate and stabilize lipophilic molecules.

Cell imaging via confocal microscopy revealed fast cell uptake of dcBSA-147-PEO(5000) $)_{28}$-(DOX) ${ }_{14}$ nanocontainers after only $1 \mathrm{~h}$ of incubation time. DOX uptake after encapsulation into micelles was fivefold increased compared to free DOX. In vitro cytotoxicity experiments revealed corresponding results since an about five-times higher cytotoxicity of dcBSA-147-PEO(5000) $)_{28}$-(DOX) $)_{14}$ micelles compared to free DOX was found. The exact mechanism of intracellular drug release is still unknown and will be investigated in future studies. The drug delivery system reported herein could in principle be used to encapsulate a broad range of lipophilic drug molecules and might therefore be attractive for combination therapy, e.g. the application of different drugs acting via different modes of action. In addition, the denatured protein backbone provides multiple functional groups available for further chemical modifications. In this way, a highly versatile and attractive platform for anticancer drug delivery was developed with great potential for in vivo studies.

Acknowledgments Financial support from NUS start-up grant under Grant No. WBS-R143-000-393-646 and WBS-R143-000-367133 and the Singapore National Research Foundation proof-of-concept Grant NRF2009-POC001-045 are greatly acknowledged.
Open Access This article is distributed under the terms of the Creative Commons Attribution License which permits any use, distribution and reproduction in any medium, provided the original author(s) and source are credited.

\section{References}

1. Davis ME, Chen Z, Shin DM (2008) Nat Rev Drug Discov 7(9):771-782

2. Kim DK, Dobson J (2009) J Mater Chem 19(35):6294-6307

3. Peer D, Karp JM, Hong S, Farokhzad OC, Margalit R, Langer R (2007) Nat Nanotechnol 2(12):751-760

4. Al-Jamal WT, Kostarelos K (2011) Acc Chem Res 44(10):10941104

5. Mikhaylov G, Mikac U, Magaeva AA, Itin VI, Naiden EP, Psakhye I, Babes L, Reinheckel T, Peters C, Zeiser R, Bogyo M, Turk V, Psakhye SG, Turk B, Vasiljeva O (2011) Nat Nanotechnol 6(9):594-602

6. Yan Z, Wang F, Wen Z, Zhan C, Feng L, Liu Y, Wei X, Xie C, Lu W (2011) J Control Release (in press)

7. Duncan R (2006) Nat Rev Cancer 6(9):688-701

8. Vaidya A, Agarwal A, Jain A, Agrawal RK, Jain SK (2011) Curr Pharm Des 17(11):1108-1125

9. Xu ZP, Zeng QH, Lu GQ, Yu AB (2006) Chem Eng Sci 61(3):1027-1040

10. Ren Y, Wong SM, Lim L-Y (2007) Bioconj Chem 18(3):836-843

11. Elzoghby AO, Samy WM, Elgindy NA (2011) J Control Release (in press)

12. Neumann E, Frei E, Funk D, Becker MD, Schrenk HH, MullerLadner U, Fiehn C (2011) Expert Opin Drug Deliv 7(8):915-925

13. Ren D, Kratz F, Wang S-W (2011) Small 7(8):1051-1060

14. Maeda H, Wu J, Sawa T, Matsumura Y, Hori K (2000) J Control Release 65(1-2):271-284

15. Lammers T, Hennink WE, Storm G (2008) Br J Cancer 99(3):392-397

16. Della Rocca J, Liu D, Lin W (2011) Acc Chem Res 44(10):957968

17. Khemtong C, Kessinger CW, Gao J (2009) Chem Commun (Camb) 24:3497-3510

18. Laginha KM, Verwoert S, Charrois GJ, Allen TM (2005) Clin Cancer Res 11(19 Pt 1):6944-6949

19. Rihova B (2009) Adv Drug Deliv Rev 61(13):1149-1158

20. Yoo HS, Lee EA, Park TG (2002) J Control Release 82(1):17-27

21. Nagykalnai $T$ (2010) Magy Onkol 54(4):359-367

22. Aluise CD, Sultana R, Tangpong J, Vore M, St Clair D, Moscow JA, Butterfield DA (2010) Adv Exp Med Biol 678:147-156

23. Lombardi G, Zustovich F, Farinati F, Cillo U, Vitale A, Zanus G, Donach M, Farina M, Zovato S, Pastorelli D (2011) Cancer 117(1):125-133

24. Gillies ER, Fréchet JMJ (2005) Drug Discov Today 10(1):35-43

25. Jain NK, Gupta U (2008) Exp Opin Drug Metab Toxicol 4(8): $1035-1052$

26. Theodore Peters J (1996) All about albumin: biochemistry, genetics and medical applications. Academic Press, San Diego and London

27. Montana M, Ducros C, Verhaeghe $\mathrm{P}$, Terme $\mathrm{T}$, Vanelle $\mathrm{P}$, Rathelot P (2011) J Chemother 23(2):59-66

28. Russell C (2011) Clin Adv Hematol Oncol 9(6):469-471

29. Yamamoto Y, Kawano I, Iwase H (2011) Onco Targets Ther 4:123-136

30. Wu Y, Chakrabortty S, Gropeanu RA, Wilhelmi J, Xu Y, Er KS, Kuan SL, Koynov K, Chan Y, Weil T (2010) J Am Chem Soc 132(14):5012-5014 
31. Zöphel L, Eisele K, Gropeanu R, Rouhanipour A, Koynov K, Lieberwirth I, Müllen K, Weil T (2010) Macromol Chem Phys 211(2):146-153

32. Eisele K, Gropeanu R, Musante A, Glasser G, Li C, Müllen K, Weil T (2010) Macromol Rapid Commun 31(17):1501-1508

33. Eisele K, Gropeanu RA, Zehendner CM, Rouhanipour A, Ramanathan A, Mihov G, Koynov K, Kuhlmann CRW, Vasudevan SG, Luhmann HJ, Weil T (2010) Biomaterials 31(33):8789-8801
34. Ritz S, Eisele K, Dorn J, Ding S, Vollmer D, Putz S, Weil T, Sinner E-K (2010) Biointerphases 5(3):FA78-FA87

35. Michael JG (1991) Cell Immunol 138(1):121-129

36. Junk MJ, Spiess HW, Hinderberger D (2010) Angew Chem Int Ed Engl 49(46):8755-8759

37. Kataoka K, Matsumoto T, Yokoyama M, Okano T, Sakurai Y, Fukushima S, Okamoto K, Kwon GS (2000) J Control Release 64(1-3):143-153 